\title{
Modeling and Simulation of Feedback based Linear Electromechanical actuator
}

\author{
Dr.B.Suresh Kumar ${ }^{a}$, K.Sindhu priya ${ }^{b}$, N. Naresh ${ }^{c}$, and Dr.S.Praveena ${ }^{d}$ \\ a Department of EEE, Chaitanya Bharathi Institute of Technology (CBIT), Hyderabad, India. \\ ${ }^{b}$ Department of EEE, JNTUH University, Hyderabad, India. \\ cDepartment of Mechanical engineering, Australia. \\ ${ }^{\mathrm{d}}$ Department of ECE, Mahatma Gandhi Institute of technology (MGIT), Hyderabad, India.
}

Article History: Received: 11 January 2021; Accepted: 27 February 2021; Published online: 5 April 2021

\begin{abstract}
An actuator is a device that which provides control over certain automation systems and also provides tuning in movement or positioning in various applications like manufacture of valves, motor, wastewater treatment plants, onboard electronics based on the type of configuration of type of actuators. Out of the available types of actuators, linear Electro Mechanical Actuators (EMA) is a powerful and advanced tool which provides optimal solution for the field of automation, various applications where high speed, high precision with control are required. This paper proposes to have velocity feedback system to entire function of EMA's in order to improve its functionality, and thus enhances the efficiency. This paper also proposes modeling and simulation of normal conventional linear Electro Mechanical Actuator and velocity feedback controlled EMA and compares the results in MATLAB/SIMULINK.
\end{abstract}

Keywords: control systems, Electro mechanical actuator (EMA), Feedback systems, and Velocity feedback systems.

\section{Introduction}

In the field of control system engineering and automation, actuators play a key role in positioning and controlling of systems. Though there are different types of actuators like hydraulic, pneumatic, electro hydraulic, an extensive based application of novel, and optimized electric actuators such as Electro Mechanical Actuators (EMA) (Battula, 2020) are preferred due to its various advantages like, overall weight is reduced, maintenance is simplified, which is often contaminant, flammable or polluting, can be eliminated. Of course, these types and configurations of actuators definitely gets changed based on its features like output torque, maximum holding force, maximum speed, ration of protection, size and type of application required.

An Electro Mechanical Actuator is a device which converts electrical energy or force into mechanical unit like kinetic energy in the form of either linear or rotary motion. Basically, the subsystem of an EMA includes power electronic circuitry, intelligent controller, an BLDC drive, ball screw mechanism for position detection, and its associated components. An intelligent controller is a PD Controller connected with feedback loop (Bennouna .O, 2013), and gives a signal comparing positional feedback signal with the reference one. A power electronic circuitry consists of conventional three phase inverter after detailed investigation on various configurations. The most critical component here is an electric drive which is a Brushless DC motor (BLDC). As BLDC is economical and has no maintenance cost like replacement of brushes, no sparking problems and less scope of commutation. So, BLDC is mostly preferred in many of the actuators due to its robustness. Choosing of this drive is important and depends upon the specific application such as stepper motor is preferred in the applications like instrumentation of robotics, laser equipment and for better accuracy. Similarly, Induction motor is chosen where accuracy and high resolution is required such as refinery process. A dc brushless motor is preferred in the flight control systems, aviation, navigation purposes. In this paper, a normalized dc motor (Garikapati, P, 2020) is taken into account as it is simple for the sake of analysis purposes, tuning of motor parameters gives clear illustration in the SIMULINK.

\section{Feedback based Electromechanical actuator}

The most important parameters in EMA are desired frequency response, stability and phase lag control. The controller is PD controller as this type of controller is enough to explain the feedback system. Anyhow, if PID controller is taken, the tuning parameter of integral parameter becomes

bit difficult and not recommended if this linear EMA is extended to various applications like ship propulsion systems, air flight control systems. A PD controller is preferred after investigation of different controllers and its tuning parameters. Generally, most of the EMA does not include any feedback systems except positional feedback due to the consequences in the sizing of actuator and its nozzle. The feedbacks (Ivana Todiac 2013; Latchoumi, 2018) to the control electric drives may be position closed loop control for tuning damping forces to improve desired frequency response of actuation system, differential pressure feedback, which is mostly used in the electro hydraulic actuators to avoid vibration level. A gear has capability to decrease the motor angular speed (RPM) and increase its torque to desired values. A network of sensors used to close the feedback rings (current, angular speed and position) that control the whole actuation system. 
Taking all constraints and parameters of EMA into account, and in order to change the dynamics of actuator system, bandwidth of EMA, and as the main foremost goal is to get optimal phase lag, good frequency response system, stability, velocity feedback control (Latchoumi, 2016) is preferred after careful investigation on various feedback control systems. This paper presents the results in MATLAB/SIMULINK without and with velocity feedback systems and the basic block diagram of Electro Mechanical Actuator with position and velocity feedback is shown in the fig. 1 .

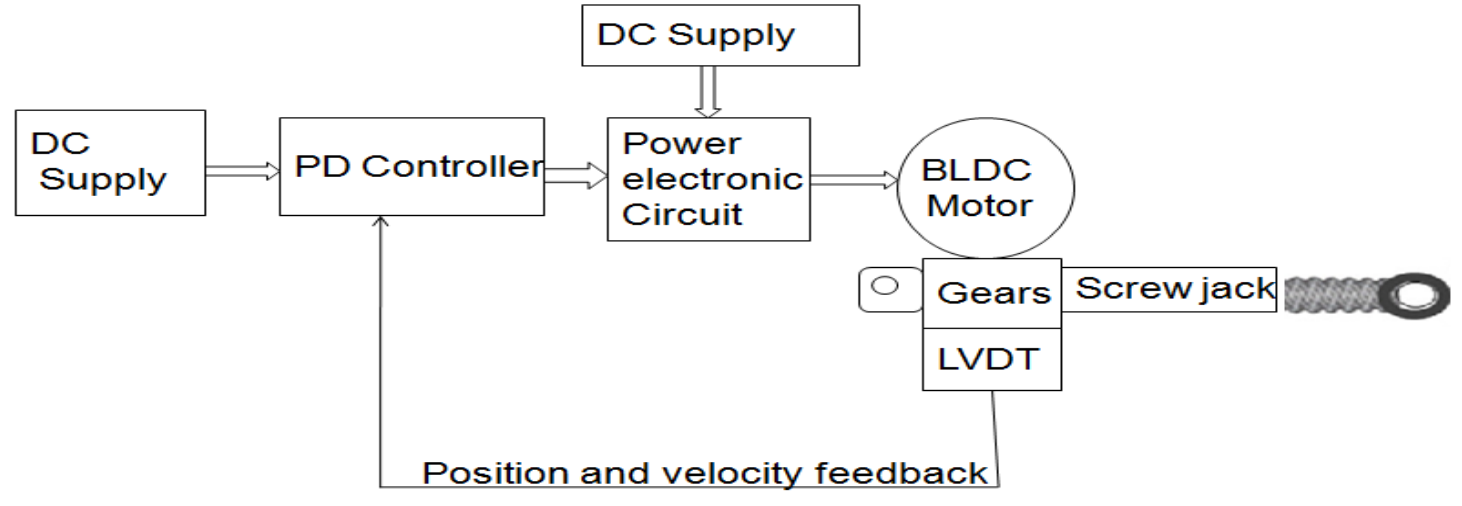

Figure.1 Block diagram of Electro Mechanical Actuator

\section{Control of EMA}

The controlling of EMA is done by mainly positional and speed regulators. Generally, the pulse width modulation technique in three phase power inverter is used to control dc motor drive for various applications of EMA. The signals from power inverter is generated based on the servo controller design i.e., PD controller based on feedback signals and reference signals. The generated signals drive the motor under different voltages and different payloads if it is application specific. According to the different voltages and input signals makes the motor rotates in clockwise and anti clock wise directions and the screw jack, LVDT sensors are connected to the shaft of motor in order to convert electrical rotations into the position. Here, in this paper, the speed or velocity of motor is taken as feedback including position and the signals of servo controller, power inverter get modified.

\section{Mathematical Modeling of DC motor}

The detailed approximated equations d-q transform is discussed in paper (Latchoumi, 2016). The equivalent electrical circuit is shown in the figure below.

Equivalent of the motor armature coil is indicated by an inductance (La) which is in series with armature resistance

( $\mathrm{Ra}$ ) along with a induced voltage ( $\mathrm{Va}$ ) which is opposing the voltage source.

Mathematically, the differential equation for the motor equivalent circuit can be designed by using basic Kirchoff's voltage law in the electrical loop.

$$
\mathrm{Va}=\mathrm{IaRa}+\mathrm{Ladi} / \mathrm{dt}+\mathrm{eb}
$$

On applying laplace transformation for the equation above,

$$
\mathrm{I}(\mathrm{s})=(\mathrm{Ia}(\mathrm{s})-\mathrm{eb}(\mathrm{s})) /(\mathrm{Ls}+\mathrm{Ra})
$$

The generated electromagnetic torque in machine is directly proportional to the armature current and can be written as

$$
\mathrm{Te}=\mathrm{KtIa} \text { and } \mathrm{Te}=\mathrm{Jdw} / \mathrm{dt}+\mathrm{B} \omega
$$

Where,,$\omega=\mathrm{d} \theta / \mathrm{dt}$

On Laplace transform,

$$
\omega(\mathrm{s})=\mathrm{Te} /(\mathrm{J} s+\mathrm{B})
$$

Where, Kt is Torque constant and $\mathrm{J}$ is inertia of motor. 
From the above equations, a dc motor is mathematically designed according to the desired application and the figure is as shown below fig. 2 .

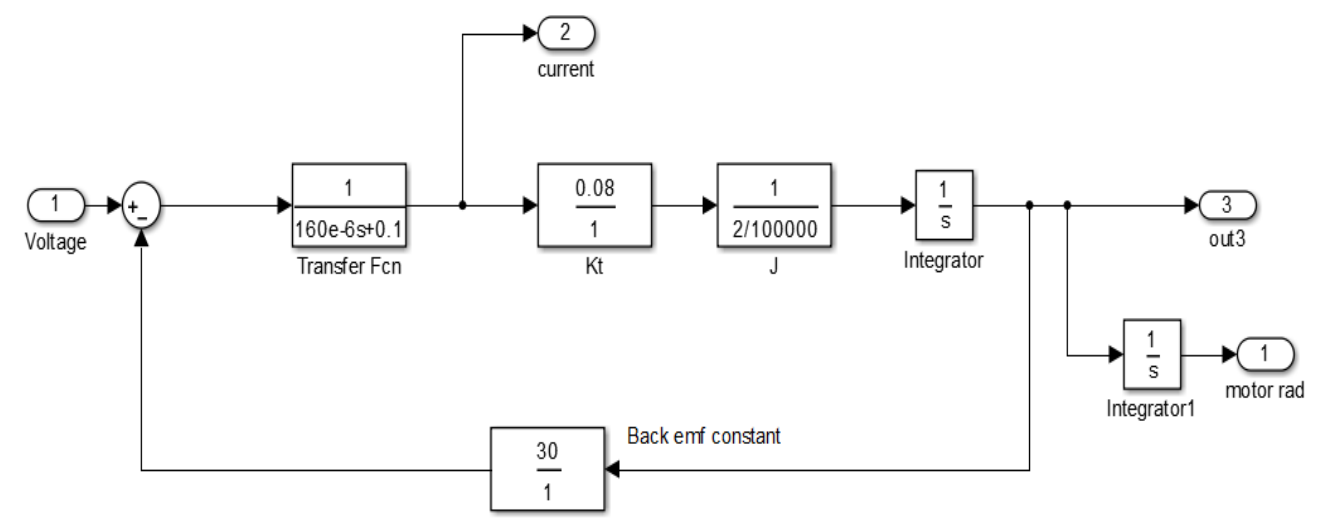

Figure.2 Mathematical model of DC motor

\section{Simulink Diagram of EMA without and with Feedback}

Fig. 3 represents an Electro Mechanical Actuator without velocity feedback i.e., the gain in the feedback path is taken as zero in the figure. As there is no feedback, there is no impact of output on input. if there is an error, motor could not follow the signal given, it may run out of undesirable path and may experience frequency related problems like peak over shoot, noise distortion which is shown in the results fig.5, fig.6, fig.7.

In the next instant add feedback parameter which is enough for the drive to follow commands given by signal generator. For instance, take the feedback parameter as 0.2 and observe in fig.8, fig. 9 .

Due to feedback provided, even there is error in the system and if motor drives out of path, the PD controller changes the signal automatically based on output desired with the help of feedback provided. The motor current and the torque can also be monitored over here but the goal of EMA is that it should follow the input command given and hence, only this part is focused in the paper by giving different types of basic signals like sinusoidal and square wave. The outputs are very smooth and accurately following the input signal. These type of systems are mostly used in targeting the objects, tracking accurately.

Table-1 Simulation parameters

\begin{tabular}{|c|c|}
\hline Rating of DC motor & $10 \mathrm{Kw}$ \\
\hline Voltage & 100 volts \\
\hline Current & $50 \mathrm{Amp}$ \\
\hline Inductance & $160 \mu \mathrm{H}$ \\
\hline Armature Resistance & $0.1 \mathrm{ohms}$ \\
\hline Torque Constant & 0.08 \\
\hline Torque & $8 \mathrm{Nm}$ \\
\hline Inertia J & $2 \times 10-5 \mathrm{~kg}-\mathrm{m} 2$ \\
\hline
\end{tabular}




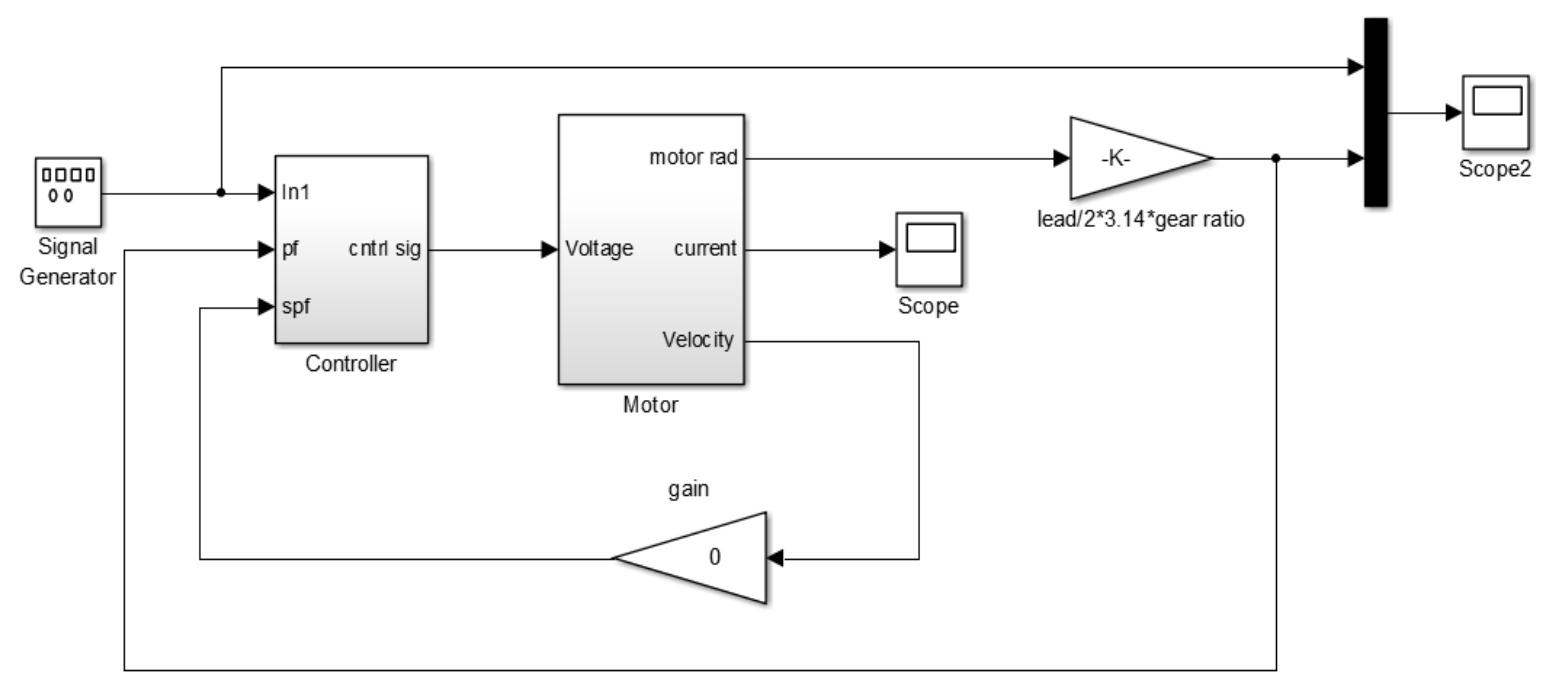

Figure.3 Simulation diagram of EMA without feedback

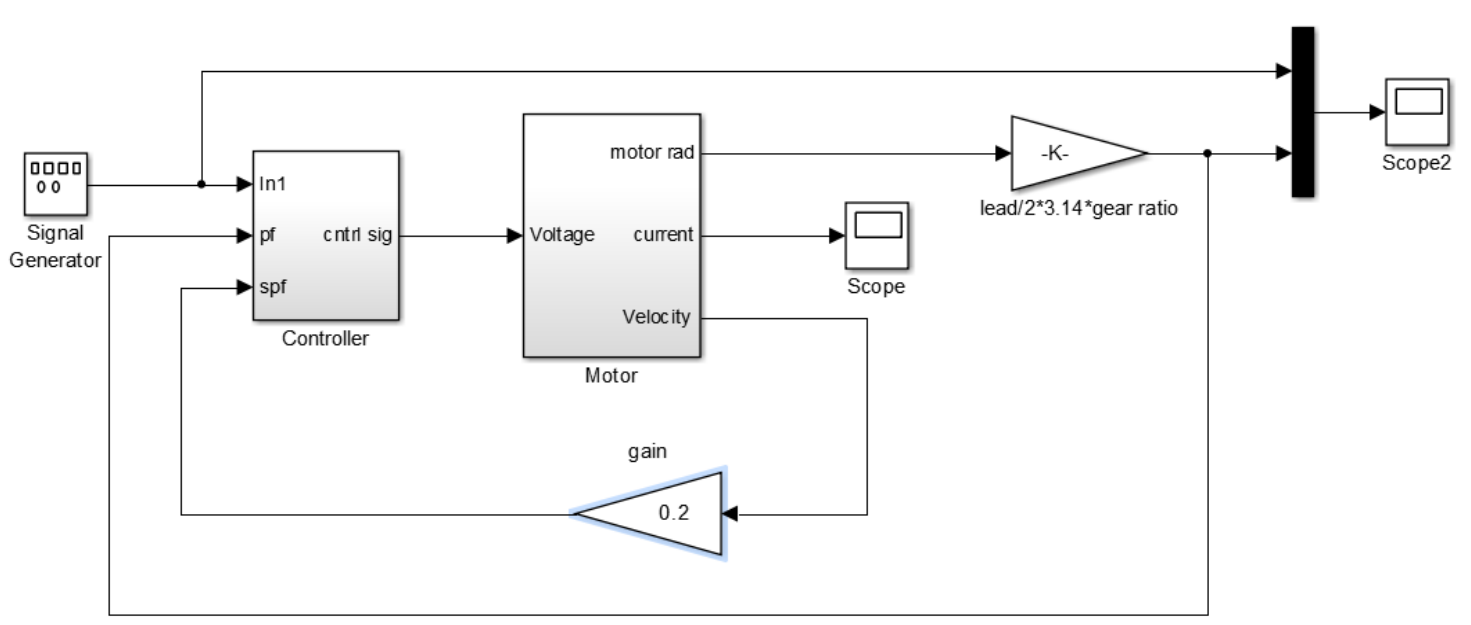

Figure.4. Actuation system with velocity feedback

The chosen parameters which are used in the simulation of this paper have been presented in the following tabular column Table-1.

\section{Simulation Results}

The simulation results for different types of input commands are shown in this section. When step command is given without any velocity feedback, the system experiences overshoot and distortions in the output as shown in Fig.5. The peak overshoot and rise time of the system is high which results in non linearity's (Ramakrishna P, 2013) in the system as shown in Fig.6. Similarly, when the sinusoidal frequency of input command increases to 25HZ, amplitude and phase are distorted. As the frequency still increases, phase lag reaches to 90 degrees as shown in Fig.7. The reason is when input amplitude is vibrating fast; the output cannot be in reach with command signal due to phase lag.

By incorporating velocity feedback in the actuation system, the overshoot and rise time is decreased when compared to without feedback as shown in Fig.8. One of the possible solutions is to add an acceleration loop and try to eliminate error in steady state (Murugan, S., 2020; Narayana P, 2016). The feedback provided here is to give acceleration to drive the motor for required output. It acts as damping to the system by controlling the speed response. To remove phase lag between input command and output signal, reducing the servo loop gains is the standard procedure to stop the oscillations. The velocity loop gain is adjusted such that the non linearity's in the system should be eliminated and to ensure the smooth operation of the system as shown in Fig.9. 


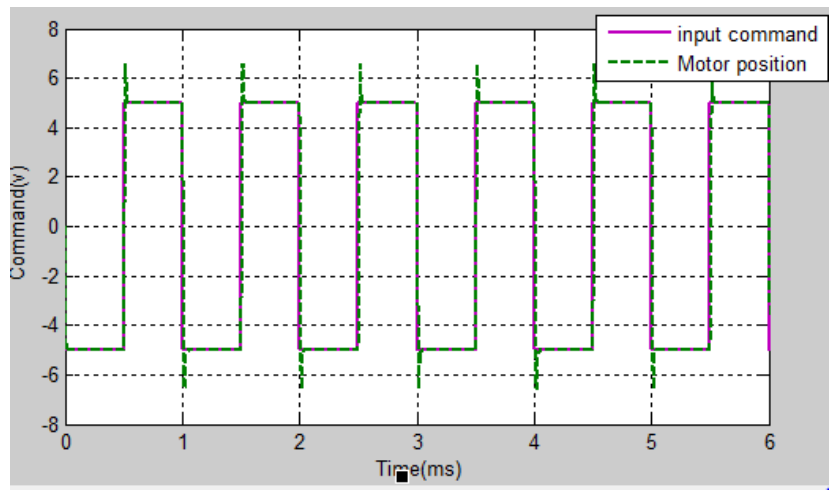

Figure.5 output of actuator with step command and without velocity loop

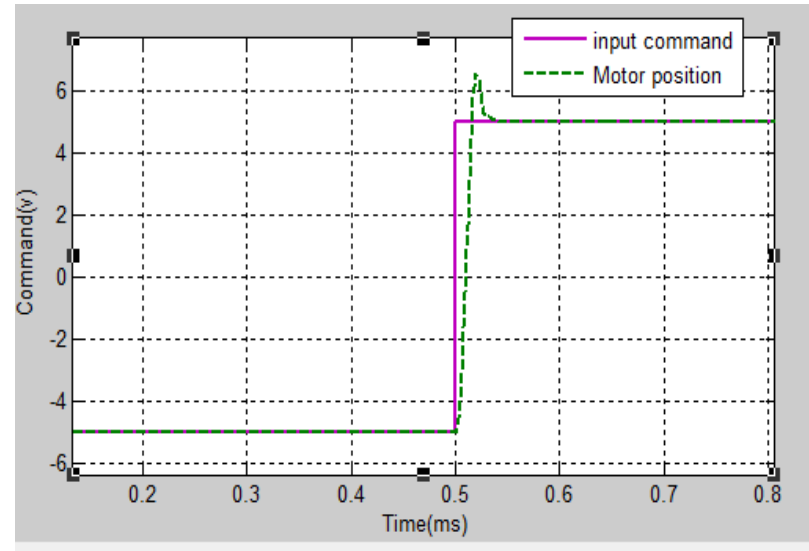

Figure.6 Peak overshoots in square signal

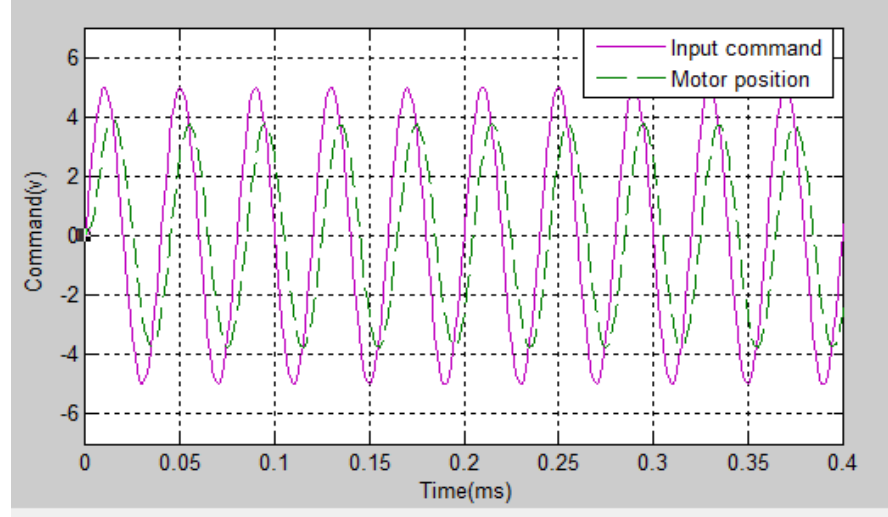

Figure.7 Output of actuator with sine command and without velocity loop

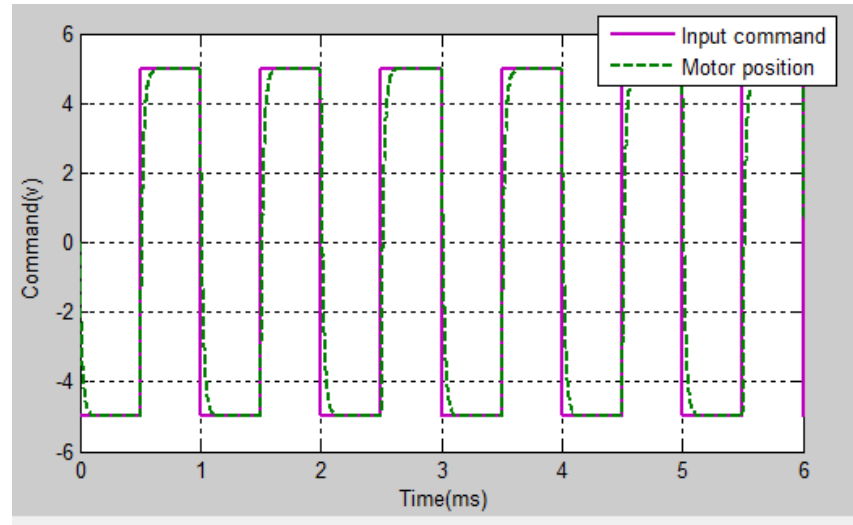

Figure.8 Output of actuator with square command with velocity loop 


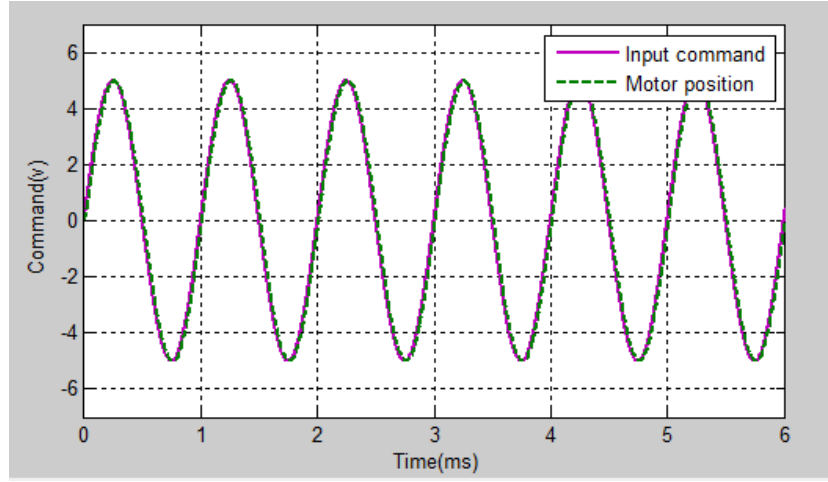

Figure.9 Output of actuator with sine command with velocity feedback

\section{Conclusion}

On simulating Electro Mechanical Actuator (EMA) model with the implementation of velocity feedback system, the performance parameters of control systems engineering like stability, peak overshoot, rise time, phase lag are improved. Improvements in these parameters in the system give reliability and smooth operation in actuation systems which are having enormous applications (Sampathkumar 2020; Shankar, 2020) in almost every field of engineering (Sureshkumar, 2019; Vasanth, 2018).

\section{References}

1. Battula, B., Anusha, V., Praveen, N., Shankar, G., \& Latchoumi, T. P. (2020). Prediction of Vehicle Safety System Using Internet of Things. Journal of Green Engineering, 10(4), 1786-1798.

2. Bennouna .O, N.langlois, (2013). "Modeling and simulation of Electromechanical Actuator for aircraft nacelles" 9th International symposium on Mechtronics and its applications. "Dynamics of motor with velocity feedback"-Massachusetts institute of technology, Department of electronics engineering.

3. Garikapati, P., Balamurugan, K., Latchoumi, T. P., \& Malkapuram, R. (2020). A Cluster-Profile Comparative Study on Machining AlSi 7/63\% of SiC Hybrid Composite Using Agglomerative Hierarchical Clustering and K-Means. Silicon, 1-12.

4. Ivana Todiac, Marko molic, (2013). "Position and speed control of Electromechanical Actuator for aerospace application”.

5. Jean-claude Derrian, Sagem defensesecurite," ElectroMechanical Actuator for advanced Technoilogies for flight controls", 28th international congress of the aeronauticalsciences.

6. Latchoumi, T. P., \& Parthiban, L. (2018). Advanced Overlap Community Detection by Associative Rule Mining and Multi-View Ant Clustering. International Journal of Engineering and Technology, 7, 21-29.

7. Latchoumi, T. P., Loganathan, J., Parthiban, L., \& Janakiraman, S. (2016, August). OFS method for selecting active features using clustering techniques. In Proceedings of the International Conference on Informatics and Analytics (pp. 1-4).

8. Latchoumi, T. P., \& Parthiban, L. (2016). Secure Data Storage in Cloud Environment using MAS. Indian Journal of Science and Technology, 9, 24-29.

9. Pillay .P and R. Krishnan ," Modelling of permanent magnet motor drives “-IEEE transactions Ind. Electrical technology.

10. Ramakrishna P., Varghese, S., Soni, N. L., \& Patel, R. J. (2013). "The effect of velocity feedback in position control of high performance servo hydraulic actuators”. 2013 International Conference on Control Communication and Computing (ICCC). doi:10.1109/iccc.2013.673169

11. Murugan, S., Jeyalaksshmi, S., Mahalakshmi, B., Suseendran, G., Jabeen, T. N., \& Manikandan, R. (2020). Comparison of ACO and PSO algorithm using energy consumption and load balancing in emerging MANET and VANET infrastructure. Journal of Critical Reviews, 7(9).

12. Narayana P mahajan, International journal of scientific and research publications. "Study of non-linear Behaviour of DC motor Using modeling and simulation"

13. Sampathkumar, A., Murugan, S., Sivaram, M., Sharma, V., Venkatachalam, K., \& Kalimuthu, M. (2020). Advanced Energy Management System for Smart City Application Using the IoT. In Internet of Things in Smart Technologies for Sustainable Urban Development (pp. 185-194). Springer, Cham.

14. Sampathkumar, A., Murugan, S., Rastogi, R., Mishra, M. K., Malathy, S., \& Manikandan, R. (2020). Energy Efficient ACPI and JEHDO Mechanism for IoT Device Energy Management in Healthcare. In Internet of Things in Smart Technologies for Sustainable Urban Development (pp. 131-140). Springer, Cham. 
15. Shankar, G., Latchoumi, T. P., Chithambarathanu, M., Balayesu, N., \& Shanmugapriya, C. (2020). An Efficient Survey on Energy Conservation System with Video Surveillance. Journal of Xian University of Architecture and Technology, 12(7), 100-106.

16. Suresh Kumar B., Ravi Kumar B.V., Sindhu Priya K. (2019) "Modeling and Simulation of Dual Redundant Power Inverter Stage to BLDCM for MEA Application”. In: Saini H., Singh R., Kumar G., Rather G., Santhi K. (eds) Innovations in Electronics and Communication Engineering. Lecture Notes in Networks and Systems, 65. Springer, Singapore.

17. Suresh kumar .B, Dr.S.Praveena, K.Sindhu priya, (2020). "Modelling and simulation of linear electromagnetic actuator for missile application with high redundancy", Mukt Shabd Journal, 9(4).

18. Vasanth, V., Venkatachalapathy, K., Thamarai, L., Parthiban, L., \& Ezhilarasi, T. P. (2017). A survey on cache route schemes to improve QoS in AD-HOC networks. Pakistan Journal of Biotechnology, 14, 265-269.

19. Vasanth, A. V., Venkatachalapathy, K., Latchoumi, T. P., Parthiban, L., Sowmia, T., \& OhmPrakash, V. (2018). An Efficient Cache Refreshing Policy to Improve QoS in MANET Through RAMP. In Proceedings of the Second International Conference on Computational Intelligence and Informatics (pp. 369-381). Springer, Singapore. 